\title{
Cooperative scale-free networks despite the presence of defector hubs
}

\author{
J. Poncela ${ }^{1}$, J. Gómez-Gardeñes ${ }^{1,2(a)}$, L. M. Floría ${ }^{1,3}$, Y. Moreno ${ }^{1,4}$ and A. Sánchez ${ }^{1,5,6}$ \\ ${ }^{1}$ Institute for Biocomputation and Physics of Complex Systems (BIFI), University of Zaragoza \\ E-50009 Zaragoza, Spain, EU \\ ${ }^{2}$ Departamento de Matemática Aplicada, ESCET, Universidad Rey Juan Carlos \\ E-28933 Móstoles (Madrid), Spain, EU \\ ${ }^{3}$ Departamento de Física de la Materia Condensada, Universidad de Zaragoza - E-50009 Zaragoza, Spain, EU \\ ${ }^{4}$ Departamento de Física Teórica, Universidad de Zaragoza - E-50009 Zaragoza, Spain, EU \\ ${ }^{5}$ Grupo Interdisciplinar de Sistemas Complejos (GISC), Departamento de Matemáticas, \\ Universidad Carlos III de Madrid - E-28911 Leganés (Madrid), Spain, EU \\ ${ }^{6}$ Instituto de Ciencias Matemáticas, CSIC-UAM-UC3M-UCM-E-28006 Madrid, Spain, EU
}

PACS 89.75.Fb - Structures and organization in complex systems

PACS $87.23 . \mathrm{Kg}$ - Dynamics of evolution

PACS 89.65.-s - Social and economic systems

\begin{abstract}
Recent results have shown that heterogeneous populations are better suited to support cooperation than homogeneous settings when the Prisoner's Dilemma drives the evolutionary dynamics of the system. The same occurs when the network growth is coevolving together with the evolutionary dynamics, which also gives rise to highly cooperative scale-free networks. In the latter case, however, the organization of cooperation is radically different with respect to the case in which the underlying network is static. In this paper we study the structure of cooperation in static networks grown together with evolutionary dynamics and show that the general belief that hubs can only be occupied by cooperators does not hold. Moreover, these scale-free networks support high levels of cooperation despite having defector hubs. Our results have several important implications for the explanation of cooperative behavior in scale-free networks and highlight the importance that the formation of complex systems have on its function.
\end{abstract}

Evolutionary dynamics [1] has attracted a lot of interest in the physics community lately, in particular in the context of evolutionary games on graphs [2,3]. This is a most relevant problem both from the physics viewpoint as well as from its applications. Indeed, evolutionary games describe a local optimization dynamics, which is largely different from the hamiltonian dynamics that is the traditional physics paradigm. On the other hand, these problems are related to important biological and socioeconomical issues, such as the emergence of cooperation [4].

To date, a great deal of work has been done on evolutionary game dynamics on fixed networks (see, e.g., [2] and references therein). Beginning with the pioneering work by Nowak and May [5], much research has focused on whether the chances of establishing cooperative behavior (if not global, at least to a large extent) are improved by

\footnotetext{
(a) E-mail: gardenes@gmail.com
}

the existence of a network (the hypothesis of network reciprocity [6]). In the case of degree homogeneous networks (such as, e.g., lattices, or Erdős-Renyi random graphs), there have been several contradictory results. For instance, it was recently reported that lattices inhibit cooperation in Snowdrift games [7] but also that they enhance it [8]. In fact, both reports are correct in so far as, as has been shown recently (see $[3,9]$ and references therein), the outcome of evolutionary games on these graphs is largely dependent on the model details (such as evolutionary update rule, network clustering, etc.). For degreeheterogeneous networks, and in particular for scale-free (SF) networks [10], there are grounds to claim an improvement of cooperation at least in some parameter regions [11-15]. The reason behind the increase of cooperation levels in scale-free networks is that hubs are occupied by cooperators, which ensures their long term success and higher levels of cooperation in the network. In fact, 
the probability that a node of degree $k$ plays as a cooperator increases with $k$ [16], which leads to an organization of cooperation in scale-free networks radically distinct from that of homogeneous topologies [11].

In all the aforementioned cases, the studied networks are static, and taken as given from the start. Other papers have considered networks co-evolving with the strategies (see, e.g., [17-24]) but co-evolution took place from an initial network where all the participating individuals were already present when the process began. Therefore, the question as to the origin of networks that support cooperative behavior remained largely unanswered. On the other hand, it would also be important to elucidate whether playing while growing is qualitatively and quantitatively equivalent to play once the networks are grown and to test the validity of phenomenological arguments in the new, more complex, dynamical scenario. We note that this issue is relevant in the wider context of complex adaptive systems, namely the debate "form follows function" or "function follows form", which arises in many situations of interest.

To answer the previous questions, we have recently [24] introduced a self-organized network model and shown that when networks grow coevolving with the strategies of the individuals that take part on them, robust, highly cooperative behavior also arises. More importantly, it was also shown that such a growth dynamics gives rise (for certain parameters, see below) to scale-free networks that supported higher cooperative levels than the BarabásiAlbert ones. In this paper, we focus on a different aspect, namely, on the microscopic interplay between structure and dynamics that gives rise to the enhancement of cooperation as well as on its novel features. Specifically, our results show that the statement that hubs are always occupied by cooperators, is no longer true under coevolution of network growth and strategies. Therefore, we report on the structural and dynamical mechanisms allowing that defector hubs can also be asymptotically stable in a network sustaining high levels of cooperation. This feature was already anticipated in [25], where by means of a toy model it was analytically shown that under certain conditions, it is possible to design heterogeneous networks in which cooperator and defector hubs coexist in the system. Here we show that structures similar to the theoretically proposed ones can and do arise in self-organized, co-evolutionary networks and play a key role in governing their properties.

Let us first describe the model of growing network used henceforth [24]. As noted above, we change focus to the long time dynamics of the strategists, in order to discover the reasons for the well suitableness of the evolved networks to support cooperation. Our model is defined as follows: beginning with an initial complete graph of $m_{0}$ nodes, new elements are sequentially attached to the network following a preferential attachment rule that depends on the payoffs of a Prisoner's Dilemma (PD) game [26]. In this game, mutual cooperation (C) provides the players with the reward payoff $R$, whereas mutual defection (D) yields them the punishment payoff $P$. An encounter between a cooperator and a defector leads to the sucker's payoff $S$ and the temptation payoff $T$, respectively. We work here in the weak $\mathrm{PD}$ regime, with parameters $T=b>R=1>P=S=0$. The network growth process consists then of an evolutionary game dynamics coupled to an attachment process. Evolutionary game dynamics proceeds as follows: at equally spaced time intervals $\tau_{D}$ every node plays a PD with her neighbors and collects the corresponding payoffs. Subsequently, players update their strategy according to the following rule $[11,13]$ : every node chooses randomly a neighbor, say $j$, and, if the chosen neighbor's payoff, $f_{j}(t)$, is larger than her own $f_{i}(t)$, she copies her neighbor's strategy with probability

$$
P_{i}=\frac{f_{j}(t)-f_{i}(t)}{b \max \left[k_{i}(t), k_{j}(t)\right]},
$$

where $k_{i}(t)$ is the degree of node $i$ at time $t$. The above updating rule prevents that agents adopt a strategy performing worse than theirs. However, if agents are allowed to make the latter irrational moves the average level of cooperation observed during network growth decreases [27] as shown also for static networks in [28]. On the other hand, the attachment process is as follows: at equally spaced intervals $\tau_{T}$, newcomers are incorporated in the network by setting up $m$ links, with probability given by

$$
\Pi_{i}(t)=\frac{1-\epsilon+\epsilon f_{i}(t)}{\sum_{j=1}^{N(t)}\left(1-\epsilon+\epsilon f_{i}(t)\right)},
$$

where $N(t)$ is the size of the network at the considered instant, and $\epsilon$ controls the strength of the game contribution to evolution, $\epsilon \rightarrow 0$ and $\epsilon \rightarrow 1$ (see footnote ${ }^{1}$ ) being the weak and strong selection limits, respectively. Thus, nodes with larger payoffs are more often chosen for linking by the newcomers, a mechanism we call evolutionary preferential attachment (EPA).

Our object of study in this paper corresponds to the case $^{2}$ when $\tau_{D} / \tau_{T}=10$, i.e., network growth is faster than game dynamics, and with strong selection, $\epsilon \rightarrow 1$. As was shown in [24], small values of $\epsilon$ give rise to homogeneous networks, the degree distribution becoming more and more long ranged with increasing $\epsilon$ and finally turning into scale-free networks when $\epsilon \rightarrow 1$ (see figures and discussion in [24]). In what follows, we will focus on

\footnotetext{
${ }^{1}$ Note that the strong selection limit is only defined approaching 1 from the left and excluding $\epsilon=1$.

${ }^{2}$ The topological and dynamical properties of the networks generated using this recipe depend weakly on the specific choice of $\tau_{D} / \tau_{T}$, at least when $\tau_{D} / \tau_{T}>1$. Similar qualitative results are obtained using $\tau_{D} / \tau_{T}=1,5,10$, and 20. The choice of $\tau_{D} / \tau_{T}<1$ leads to a situation in which cooperation and defection cannot coexist as the system dynamics evolves either to an all-C or to all$\mathrm{D}$ configuration. Other rules for nodes attachment and evolutionary dynamics might change both the topological and dynamical features of the system [27]. We stress that our main goal is to show that there are networks for which defector hubs can be asymptotically stable, for which it is enough to find one network generation recipe.
} 


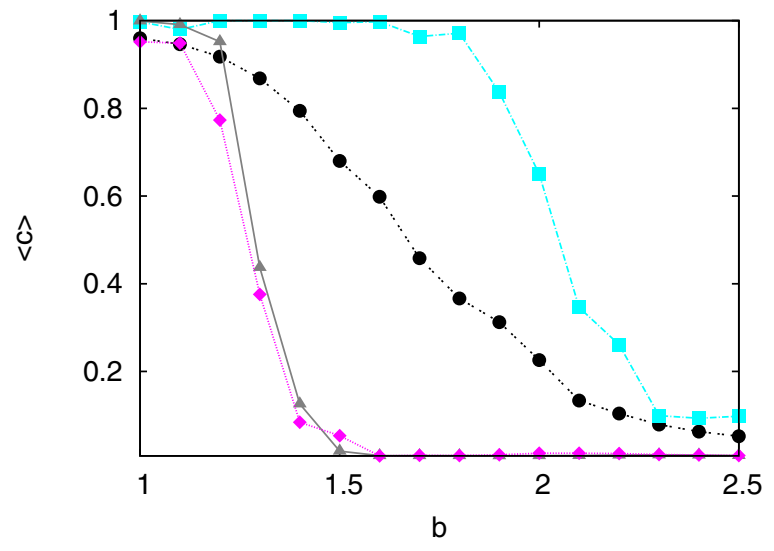

Fig. 1: (Colour on-line) Cooperation levels at the end of the growth process and after letting the network relax as a function of $b$. The original network was grown up to 4000 nodes with $\epsilon=0.99$ and the asymptotic cooperation levels are computed $10^{7}$ time steps afterwards. The networks generated are scalefree networks with an average degree $\langle k\rangle=4$. Full circles show the cooperation level when the network stops growing. The other curves show the asymptotic cooperation when the network has been randomized (triangles), when the strategies have been randomized (squares) and with both randomizations (diamonds).

the structure of the cooperative organization rather than on the structure of the network itself since we will consider the already grown graph following [24] as the starting point of our numerical experiments. As a reference, fig. 1 shows the average cooperation level in the steady state of the network resulting from our model for different values of the temptation parameter $b$ (full circles), where it can be seen that the cooperation level is quite large even for large $b$.

In order to gain more insight in the relation between network topology and the supported level of cooperation, we studied how strategies evolve after the attachment process is stopped. To this end, in fig. 1, we also show the asymptotic behavior of the network when it is randomized by reshuffling its links while preserving the degree of each node (network randomization) and when the network is kept constant but the strategies of the nodes are reassigned at random, i.e. assigning nodes' strategies with a probability equal to the final density of cooperators (strategy randomization). As can be seen from the plot, the responsible for the cooperation increment is the network structure, as its randomization leads to a decrease of cooperation at levels far away from those of the original one or even of a Barabási-Albert SF model network [10]. Note that strategy randomization does not prevent high levels of cooperation, thus confirming that the governing factor of the network behavior is the structure arising from the co-evolutionary process. Moreover, we will see later that the correlation between topological features of the nodes and the strategies that occupy them is high and thus destroying this correlation via the strategy

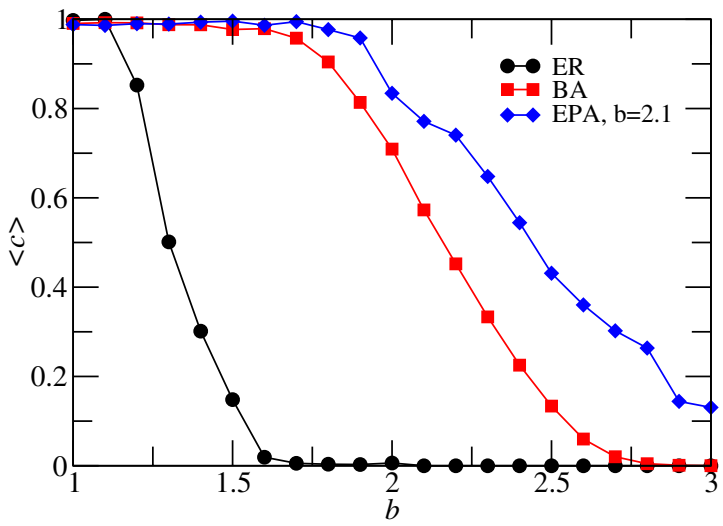

Fig. 2: (Colour on-line) Cooperation levels in Erdős-Reńyi, Barabási-Albert, and evolutionary preferential attachment network models, as a function of the temptation parameter $b$. The EPA network is built up using the model described in the main text for $b=2.1$ and $\epsilon=0.99$.

randomization affects strongly the asymptotic level of cooperation. As further evidence supporting the fact that the structure of the networks supports high levels of cooperation, fig. 2 shows an example in which one of our model networks, grown with $b=2.1$, is used as a static substrate for the evolutionary game dynamics. The plot shows that these networks, resulting from the evolutionary preferential attachment process, are remarkably more favorable to cooperation than the Barabási-Albert SF networks analyzed in prior studies $[11,13,21]$.

We have further analyzed this issue in more details looking at the distribution of cooperators with a given degree. Networks are grown as discussed before, but once they reach the size $N$, the evolutionary dynamics proceeds without growth. The distribution of cooperator by degrees is calculated at two later times. The results are shown in fig. 3. In particular, we have computed the probability that a node with degree $k$ acts as a cooperator, $P_{c}(k)$ (see footnote $\left.{ }^{3}\right)$. Filled circles show the structure of cooperation for the resulting network as a function of the degree of the nodes, making it clear that the growth process leads to a concentration of cooperators at intermediate degree nodes. This comes from the fact that while the network is growing, newcomers join it with the same probability of being cooperators or defectors. In this situation, defectors have an evolutionary advantage as they get higher payoffs from cooperator newcomers. Although these cooperators would subsequently change into defectors and stop providing payoff for the original defector, the stable source of new cooperator nodes entering the network compensates for this effect. Upon stopping the growth while letting strategies continue evolving, we observe that low degree nodes

${ }^{3}$ Note that $P_{c}(k)$ is assigned a value between 0 and 1 only when nodes of degree $k$ are present in the networks. Otherwise the corresponding value of $P_{c}(k)$ does not appear in fig. 3a. Therefore, $P_{c}(k)=0$ means that there are nodes with that degree, but they are not cooperators. 

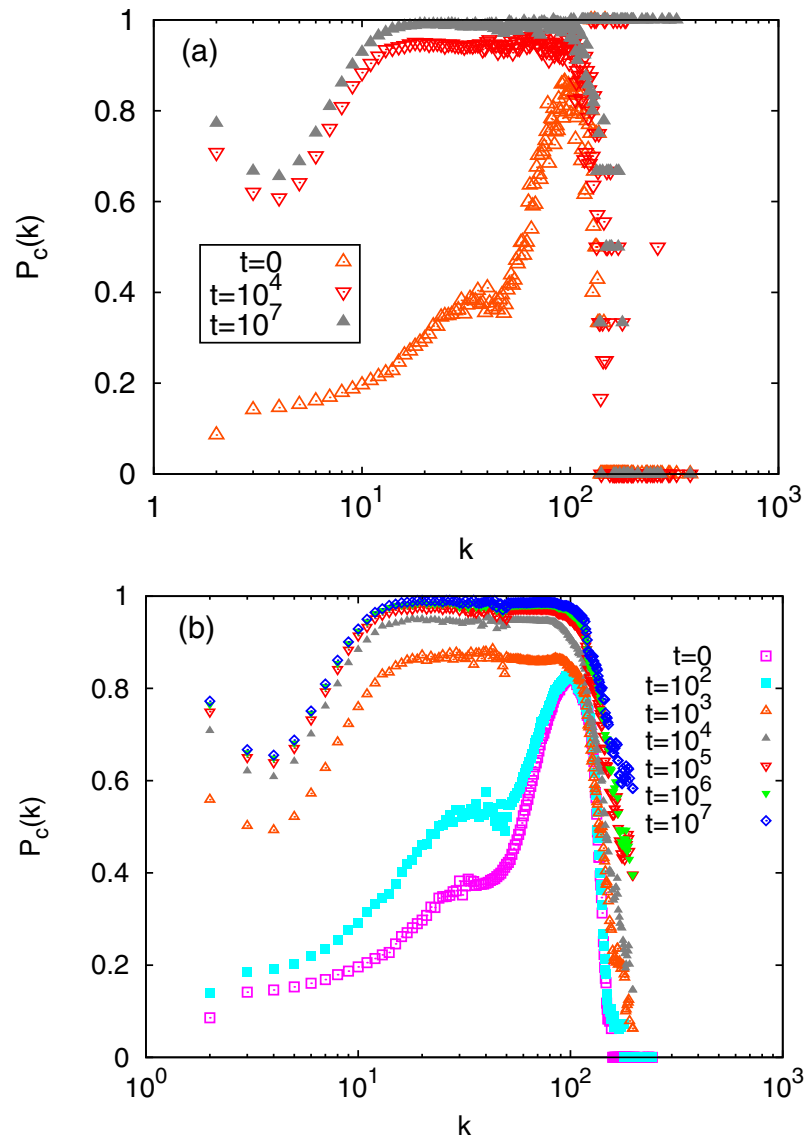

Fig. 3: (Colour on-line) (a) Cooperator distribution as a function of the degree at the end of the evolutionary preferential attachment process $(t=0), t=10^{4}$ time steps later, and $t=10^{7}$ time steps later, for $b=2.2$, as indicated. Cooperators invade almost all lowly connected nodes once the network stops growing. Note, additionally, that there are hubs playing as defectors (the ones for which $P_{c}(k)=0$ ) see footnote ${ }^{3}$. In (b) we show the running averages of the values $P_{c}(k)$ in order to remove the noise of the curves in (a). We have also included other distributions $P_{c}(k)$ taken at $t=0,10^{2}, 10^{3}, 10^{4}, 10^{5}, 10^{6}$ and $10^{7}$. From the negative slope of $P_{c}(k)$ for large values of $k$ it becomes clear that large degree individuals play as defectors with high probability.

are rapidly taken over by cooperators, and after $10^{4}$ time steps they are majoritarily cooperators. On the contrary, hubs are much more resistent to change, and even after $10^{7}$ time steps not all of them have changed into cooperators.

We now come to the main novel dynamical feature of this work. As shown in fig. 3 there are defector hubs at very long time scales. The persistence of hub defectors is a very intriguing observation, in so far as it is in contrast with the arguments in $[11,13,16]$ that show that, in scale-free networks, hubs should be cooperators (i.e., a defector hub is unstable). In a few words, this occurs because a defector sitting on a hub will rapidly convert its neighbors to defectors, which in turn leaves it with zero payoff; subsequently, if one of its neighbors turns back to cooperation, the hub will eventually follow. It seems,

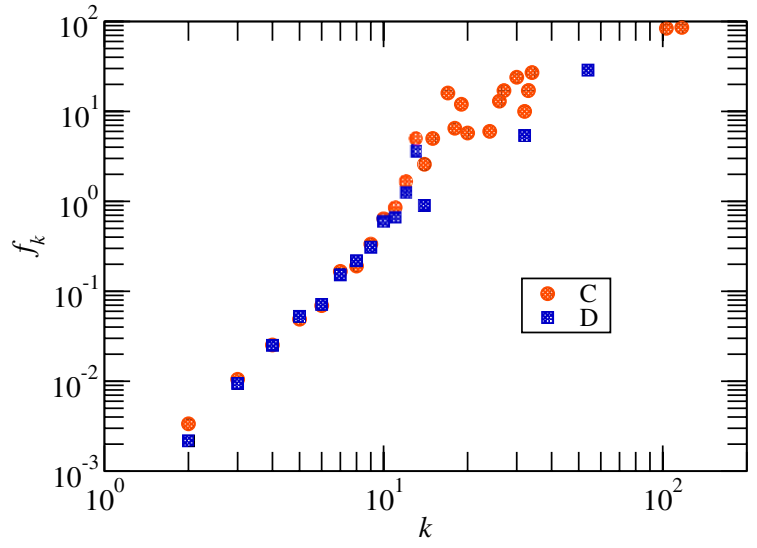

Fig. 4: (Colour on-line) Average payoffs of cooperators and defector nodes at the end of network growth $(t=0)$ as a function of their degrees, $k$, for a realization of the evolutionary preferential attachment model with $b=1.8$. Note that the similarity between cooperators' and defectors' payoffs implies that imitation events take place on a long time scale.

however, that the coupling of evolutionary game dynamics with the network growth leads to a configuration (both structural and dynamical) that stabilizes the defectors on hubs. Indeed, we have observed in our simulations that hubs are defectors for as long as evolutionary preferential attachment proceeds. The unexpected result that fig. 3 shows is that defector hubs can also be asymptotically stable once the network growth has ceased, i.e., it became static. It is important to stress that not all realizations of the process end up with defector hubs; for low values of $b$, this is practically never the case and almost no realization produces defectors at the hubs. However, as $b$ increases, the percentage of realizations where this phenomenon is observed increases rapidly.

The fact that defector hubs may have very long lives may be the relevant feature for the behavior of the network resulting from the growth process. Therefore, it is important to understand the reason for such a slow dynamics. We claim that it can be traced back to the payoff structure of the network. In fig. 4, we present an example taken from a single realization of the process. Had we plot results of payoffs averaged over realizations, we would not have been able to obtain this picture, because in that case payoffs are seemingly very different in the region of large degree, as a consequence of the statistical properties of our networks, in which hubs do exist but their degree and payoff depend on the specific realization. As can be seen, the payoff grows approximately as a power law; however, the key point here is not this law but the fact that the payoffs for defectors and cooperators of the same degree are very similar. In view of the strategy update rule, eq. (1), it becomes clear that the evolution is very slow and, if on top of that we take into account the role of the degree in that expression, we see that hubs have a very small probability to change their strategies, whatever they are. 


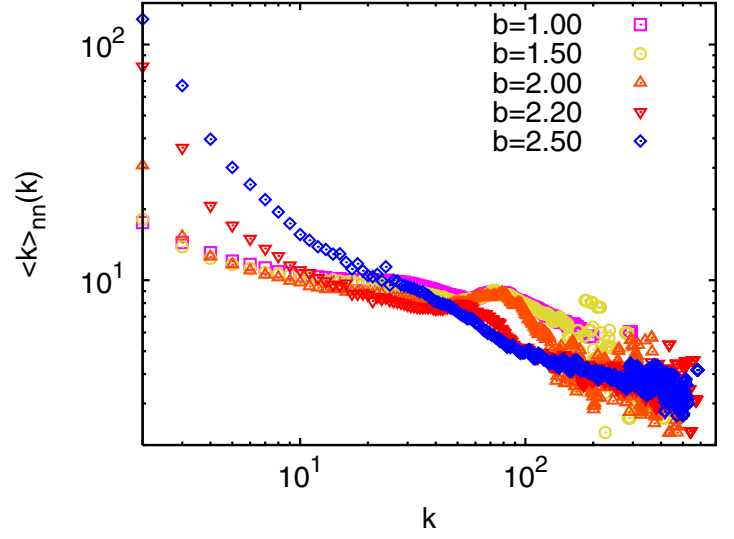

Fig. 5: (Colour on-line) Degree-degree correlations of the underlying networks. We plot the average nearest-neighbors degree $k_{n n}(k)$ of a node of degree $k$ for several values of the parameter $b$ used to generate the networks. The rest of parameters are the same used in fig. 1. Note that negative correlations imply that hubs are not likely to be connected to each other. See the text for further details.

Having identified the coexistence of cooperator and defector hubs, we next find out whether this configuration is asymptotically stable and why the hubs are not invaded by opposite strategies. The reason is rooted in the structural properties of the network: cooperator and defector hubs can coexist when they are not directly linked. This is shown in fig. 5 , where we have plotted the average degree of the nearest neighbors of a node with connectivity $k$, i.e., the degree-degree correlations of the networks. As can be seen from the figure, the networks are disassortative, i.e., with negative correlations in which highly connected nodes are more likely connected to poorly connected nodes and vice versa. Therefore, not only the hubs are not linked to each other, but they share a set of poorly connected individuals. Due to the high payoffs obtained by the hubs, the poorly connected individuals attached to the hubs experience cycles of cooperation and defection, which are, in fact, the effect of the competition for invasion among the two non-neighboring hubs. If such a local strategic configuration arises, as it is indeed the case discussed here, then neither of the two hubs will take over the set of fluctuating individuals, nor the latter will invade the hubs as they are mainly lowly connected nodes with small payoffs. This can be understood in terms of the so-called dipole model $[16,25]$, that shows analytically that two hubs with opposite strategies can coexist as we have just described. In particular, it can be argued that, in addition to fluctuating nodes, cooperator hubs should also have a set of cooperators linked to them, for this will provide the hubs with a stable source of benefits. On the contrary, defector hubs survive even if they are only connected to the set of fluctuating individuals.

In order to test if the grown networks exhibit local dipole-like structures, we have measured the connectivity of the neighbors of defector and cooperator hubs, which

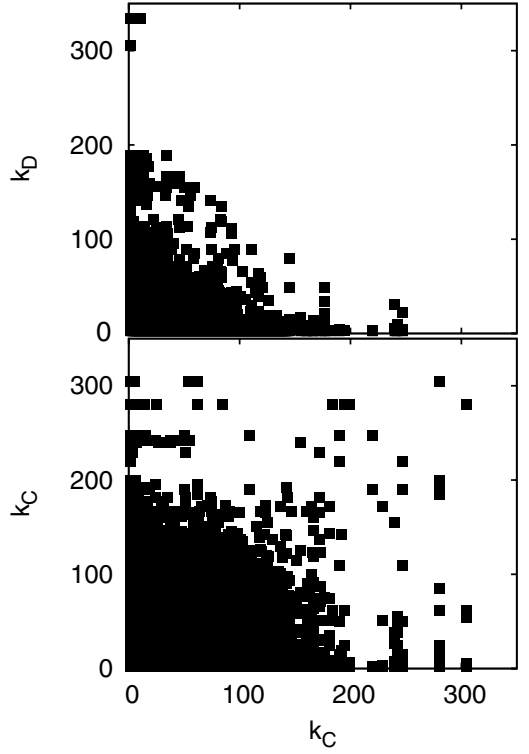

Fig. 6: Connectivity matrix of cooperators and defectors (up) and of cooperators with themselves (down). The element $(i, j)$ is set to 1 (dot in the figure) when a link between a defector (cooperator) of degree $i$ and a cooperator (cooperator) of degree $j$ exists.

we represent in fig. 6 . The figure undoubtedly shows that highly connected nodes playing as defectors are mainly connected to poorly connected cooperators (the fluctuating strategists), whereas cooperator hubs are connected to each other and also to a significant fraction of lowly connected nodes. This fully confirms that, in contrast to all previous results, there is a structure allowing the resilience of defector hubs that is similar to the dipole model.

In summary, we have shown that the coupling of evolutionary game dynamics with network growth gives rise to novel population structures on scale-free networks, characterized by the dynamic stabilization of defectors on hubs. We have shown that these defector hubs can be extremely long-lived due to the similarity of payoffs between cooperators and defectors arising from the coevolutionary process. Moreover, we have been able to link the payoff distribution to the network structure. To our knowledge, this is the first time that the payoff structure is invoked to understand evolutionary dynamics on graphs, and we envisage that its use may lead to more insights in future works. In addition, the fact that the network self-organizes creating local dipole-like structures during growth shows the relevance of the dipole model [25] to understand how other naturally occurring SF networks behave. For application to social issues, this implies that structures in which highly connected agents exploit less connected ones are dynamically possible, closely resembling real but undesirable situations such as, e.g., exploitation by a few privileged individuals or in pyramid scams. In this respect, only stopping the growth network would put an end to the exploitation process in the context of this model, and even this would not 
always be enough in view of the stability of some defector hubs. On the other hand, our simulations of the random reshuffling of strategies on the final network provide more constructive conclusions as well: in many cases, resulting networks are very supportive of cooperation and outperform other models such as, e.g., the BarabásiAlbert model. This may be of relevance to design of organizations in order to improve their performance. Finally, the phenomenon of dynamical stabilization of unstable states may be more general, and it would be worth exploring it by studying network coevolution with different dynamical systems, such as, e.g., oscillators or excitable media. This research would certainly be relevant to address the question of how physical, chemical or biological systems arise and perform.

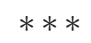

YM is supported by MICINN (Spain) through the Ramón y Cajal Programme. This work has been partially supported by MICINN through Grants FIS2006-12781C02-01, FIS2008-01240 and MOSAICO, and by Comunidad de Madrid (Spain) through Grant SIMUMAT-CM.

\section{REFERENCES}

[1] NowaK M. A., Evolutionary Dynamics: Exploring the Equations of Life (The Belknap Press of Harvard University Press, Cambridge) 2006.

[2] Szabó G. and Fáth G., Phys. Rep., 447 (2007) 97.

[3] Roca C. P., Cuesta J. and Sánchez A., Phys. Life Rev., 6 (2009) 208.

[4] Pennisi E., Science, 309 (2005) 93.

[5] Nowak M. A. and May R. M., Nature, 359 (1992) 826.

[6] NowaK M. A., Science, 314 (2006) 1560.

[7] Hauert C. and Doebeli M., Nature, 428 (2004) 643.

[8] Sysi-Aho M., SARAmäKi J., Kertész J. and KASKi K., Eur. Phys. J. B, 44 (2005) 129.
[9] Roca C. P., Cuesta J. and Sánchez A., Phys. Rev. E, 80 (2009) 046106.

[10] Bocaletti S., Latora V., Moreno Y., Chavez M. and Hwang D. U., Phys. Rep., 424 (2006) 175.

[11] Gómez-Gardeñes J., Campillo M., Floría L. M. and Moreno Y., Phys. Rev. Lett., 98 (2007) 108103.

[12] Poncela J., Gómez-Gardeñes J., Floría L. M. and Moreno Y., New J. Phys., 9 (2007) 184.

[13] Santos F. C. and Pacheco J. M., Phys. Rev. Lett., 95 (2005) 98104.

[14] Santos F. C. and Pacheco J. M., J. Evol. Biol., 19 (2006) 726.

[15] Pusch A., Weber S. and Porto M., Phys. Rev. E, 77 (2008) 036120; Assenza S., Gomez-Gardenes J. and Latora V., Phys. Rev., 78 (2008) 017101.

[16] Gómez-Gardeñes J., Poncela J., Floría L. M. and Moreno Y., J. Theor. Biol., 256 (2008) 296.

[17] Ebel H. and Bornholdt S., Phys. Rev. E, 66 (2002) 056118.

[18] Eguíluz V., Zimmermann M., Cela-Conde M. G. and San Miguel M., Am. J. Soc., 110 (2005) 977.

[19] Santos F. C., Pacheco J. M. and Lenaerts T., PLoS Comput. Biol., 2 (2006) 1284.

[20] Biely C., Dragosit K. and Thurner S., Physica D, 228 (2007) 40.

[21] Santos F. C., Pacheco J. M. and Lenaerts T., Proc. Natl. Acad. Sci. U.S.A., 103 (2006) 3490.

[22] Szolnoki A., Perc M. and Danku Z., EPL, 84 (2008) 50007.

[23] Szolnoki A. and Perc M., New J. Phys., 10 (2008) 043036.

[24] Poncela J., Gómez-Gardeñes J., Floría L. M., SÁnchez A. and Moreno Y., PLoS ONE, 3 (2008) e2449.

[25] Floría L. M., Gracia-Lázaro C., Gómez-Gardeñes J. and Moreno Y., Phys. Rev. E, 79 (2009) 026106.

[26] Rapoport A. and Chammah A. M., Prisoner's Dilemma (University of Michigan Press, Ann Arbor) 1965.

[27] Poncela J., Gómez-Gardeñes J., Traulsen A. and Moreno Y., New J. Phys., 11 (2009) 083031.

[28] Ohtsuki H. and Nowak M. A., J. Theor. Biol., 243 (2006) 86. 